\title{
REGENERACIÓN DE UN ALCORNOCAL INCENDIADO EN EL SUR DE ESPAÑA (ISTÁN. MÁLAGA)
}

\author{
Baltasar CABEZUDO, Andrés PEREZ LATORRE y José M. NIETO
}

\begin{abstract}
Resumen: Regeneración de un alcornocal incendiado en el sur de España (Istán. Málaga). Se ha realizado un estudio sobre los efectos del fuego en las especies perennes de un alcornocal ombrófilo en el sur de España. La mayoría de las especies presentan estrategias de regeneración vegetativas mediante yemas epicórmicas $(56 \%)$ y yemas no epicórmicas $(27 \%)$. Sólo un $10 \%$ presentan regeneración mediante semillas y dos especies (Abies pinsapo Boiss. yAsplenium onopteris L.) han desaparecido de la zona incendiada. La incidencia del fuego sobre el alcornocal se traduce fundamentalmente en la pérdida de individuos totales (50\%) y no en la pérdida de diversidad (2 especies). Quercus suber sobrevivió al incendio en un $46 \%$. A los tres meses del incendio, la mayoría de las especies presentaban procesos de regeneración. El pastoreo en la zona incendiada es selectivo sobre ciertas especies, e incide negativamente sobre el crecimiento global de la comunidad, sin producir efectos aparentes ni sobre el número de individuos regenerados ni sobre la pérdida de diversidad.
\end{abstract}

Palabras clave. Fuego, alcornocal, regeneración, Quercus suber, Andalucía, España.

Abstract: After fire regeneration in a Quercus suber forest in the South of Spain (Istán. Málaga). A study on the fire effects and adaptations in the perennial species of a Quercus suber forest in the South of Spain (West Mediterranean) have been carried out. Most species show vegetative after fire regeneration strategies by epicormic buds (56\%) and non epicormic buds (27\%). Only $10 \%$ species have seed regeneration and two species (Abies pinsapo Boiss. and Asplenium onopteris L.) dissapeared in the burned area. The fire effect on the burned vegetation causes, fundamentally, a loss of individuals ( $50 \%$ over the total) but not a diversity loss (only two species dissapeared). Quercus suber survived in a 50\%. After tree months from the fire, most species had regeneration dynamics. Grazing affects in a negative way the community total growth, but does not affect, outwardly, the total number of regenerated individuals and the loss of diversity.

Key words. Regeneration after fire, Quercus suber forests, Andalusia, Spain.

\section{INTRODUCCIÓN}

El alcornocal es una formación vegetal característica del Mediterráneo Occidental y que en el sur de la Península Ibérica (Andalucía) se presenta con un aceptable estado de conservación y una marcada diversidad florística y fitocenológica (Pérez Latorre $e t a l$,
1993, 1994). El fuego, factor con fuerte incidencia histórica en Andalucía, ha determinado en gran medida la estructura, composición florística y distribución actual de los alcornocales (Pons y Thinon, 1987, Naveh, 1991 ) y sus efectos han sido estudiados dentro de la península Ibérica por Souto \& Monteiro (1987) en alcornocales portugueses, Pery 
(1989) en alcornocales catalanes y García Fayos (1991) en alcornocales valencianos. Todos ellos consideran al alcornocal, al igual que el resto de la vegetación mediterránea (Le Houerou, 1981), como una formación pirófita con gran capacidad de regeneración tras el fuego, y que, después de algunos años, puede llegar a recuperar totalmente su estructura original. Esta recuperación es llevada a cabo por las distintas especies del alcornocal, mediante estrategias diversificadas que pueden favorecer a unas sobre otras, modificando con el tiempo la estructura y composición florística del mismo (Arianoutson \& Margaris, 1981; Trabaud, 1987). La capacidad de regeneración del alcornocal se modifica negativamente en función de la intensidad del fuego, de su recurrencia (Delarze et al, 1990) y por el pastoreo (Naveh, 1974, 1991).

En este trabajo se pretende determinar el comportamiento frente al fuego de las especies más importantes de un alcornocal ombrófilo andaluz en cuanto a tipos de regeneración, pérdida de diversidad específica y en el número de individuos, fenología de la regeneración y comprobar el efecto que sobre esta respuesta tiene la presión de los herbívoros domésticos y silvestres.

\section{MATERIAL Y MÉTODOS}

El alcornocal estudiado se sitúa en el Monte de Bohornoque, término municipal de
Istán (Málaga, Costa del Sol) y dentro del Parque Natural Sierra de las Nieves. El Parque Natural sufrió un incendio en Agosto de 1991 que afectó parcialmente a la masa de alcornoques que hasta ese momento presentaba un excelente estado de conservación debido a la inexistencia de fuegos recientes y a un uso racional y conservativo del mismo. Dicho alcornocal se instala sobre inceptisoles de escasa profundidad desarrollados a partir de micaesquistos, entre el piso bioclimático termomediterráneo superior y el mesomediterráneo inferior y con un ombroclima húmedo inferior (tab. 1). Fitosociológicamente se incluye en la asociación Teucrio baetici-Quercetum suberis en su variante con Abies pinsapo (Pérez Latorre et al., 1993, 1994), característica del subsector Marbellí, sector Aljíbico (Nieto et al., 1991) y es rico en especies del madroñal (CytisoArbutetum unedi).

Con el fin de detectar la fenología de la regeneración de las especies afectadas por el fuego y medir el crecimiento alcanzado durante los meses siguientes, se inició un seguimiento en el área de estudio a los cinco días del incendio; con los datos se elaboró una tabla en la que se indica la fecha de aparición del primer rebrote detectado y la altura máxima alcanzada en cada muestreo. Para cada especie se estudió la estrategia (carácer ecomorfológico) de «regeneración vegetativa tras fuego» (Orshan, 1986), estrategia ligada fuertemente con el

\begin{tabular}{lcccccccccc}
\hline Estación & $\mathrm{P}$ & $\mathrm{T}$ & $\mathrm{It}$ & $\mathrm{Ia}$ & $\mathrm{ETP}$ & $\mathrm{BA}$ & $\mathrm{EST}$ & $\mathrm{PF}$ & $\mathrm{PS}$ & Ic \\
\hline Istán & 906 & 17.1 & - & 33.5 & 863 & +43 & IOPV & $1-2$ & 3.5 & 21.3 \\
Tolox & 962 & 18.3 & 398 & 33.9 & 954 & +8 & IPOV & $1-2$ & 3.5 & 28.0 \\
Ojén (S.B.) & 1026 & 17.9 & 412 & 36.7 & 898 & +128 & IOPV & 0 & 3.5 & 18.6 \\
\hline
\end{tabular}

Tabla 1. Datos de las estaciones climáticas mas cercanas al área de estudio. $\mathrm{P}=$ precipitación media anual, $\mathrm{T}=$ temperatura media anual, It=índice de termicidad, Ia=índice de aridez de De Martonne, $\mathrm{ETP}=$ evapotranspiración potencial, $\mathrm{BA}=$ balance anual (P-ETP), EST=estacionalidad de las precipitaciones, $\mathrm{PF}=$ periodo frío de Emberger, $\mathrm{PS}=$ periodo seco, Ic=índice de continentalidad de Gorezynski. Climatic data from the nearest study area stations. $P=$ annual rainfall average, $T=$ annual temperature average, It=Rivas Martínez thermicity index, I $a=D e$ Martonne aridity index, $E T P=$ potential evapo-transpiration, $B A=$ annual balance $(P-E T P), E S T=$ rainfall seasonality, $P F=$ Emberger cold season, $P S=$ dry period, $I c=$ Gorezynski continentality index. 
comportamiento hidroecológico y biotipo de la especie (Naveh, 1991), y cuyos tipos son: regeneración por yemas epicórmicas sobre el suelo, por yemas epicórmicas bajo el suelo, yemas no epicórmicas bajo el suelo, regeneración mediante semillas y sin ningún tipo de regeneración.

Durante el año posterior al fuego, y con el objetivo de tener una estimación de la supervivencia del alcornoque (Quercus suber), se hizo un seguimiento de 100 alcornoques situados en una parcela uniformemente afectada por el fuego dentro del área de estudio. Se contabilizaron los árboles muertos, los que rebrotaron inicialmente y los que tras rebrotar se secaron. Los árboles afectados presentaban corcho de regeneración de 6 años, por lo que se encontraban, en principio, suficientemente protegidos frente al fuego en la base del tronco.

A fin de establecer la incidencia del fuego sobre la masa quemada, se analizó la diversidad y número de individuos totales y por especies en una parcela (testigo) de $200 \mathrm{~m}^{2}$ no afectada por el fuego y situada en el límite de la zona incendiada. Estos datos nos permitieron, una vez iniciada la regeneración en el área quemada, estimar la posible pérdida de diversidad específica y la pérdida de individuos por especies. El área mínima obtenida en la zona de estudio fue de $75 \mathrm{~m}^{2}$.

Ya que el pastoreo ha actuado como factor selectivo muy fuerte en los ecosistemas mediterráneos (Naveh, 1991), y con el fin de estimar la pérdida de diversidad y abundancia de las especies, así como la incidencia que sobre la regeneración pudiera tener la presión de herbívoros silvestres o domésticos, se eligieron dos parcelas en la zona quemada, una de ellas $\left(80 \mathrm{~m}^{2}\right)$ fué vallada a fín de impedir la entrada de ganado, la segunda $\left(300 \mathrm{~m}^{2}\right)$ se dejó sin vallar y libre al pastoreo. En ambas parcelas se hizo una estimación, en función del area mínima, del número de individuos totales y por especies previos al incendio. Durante un periodo de seis meses se hizo un seguimiento de la regeneración de ambas parcelas mediante la toma de los siguientes datos: numero total de individuos regenerados, numero de individuos regenerados por especies, estrategia de regeneración y altura máxima media (media de las máximas alturas de todos los individuos de cada especie).

\section{RESULTADOS}

En la tabla 2 se presentan los resultados sobre la fenología de la regeneración del alcornocal (Septiembre a Diciembre). A pesar de ser una de las épocas más desfavorables para el crecimiento vegetativo, la comunidad da muestras de actividad a los pocos días del incendio (dos-tres semanas), existiendo un retardo de la elongación en Diciembre al coincidir con el mínimo vegetativo anual de la comunidad (Pérez Latorre et al, 1995). Algunas especies, como Arbutus unedo, Erica arborea, Quercus suber, Ulex baeticus, etc., presentan procesos de regeneración inmediatamente después del incendio. Otras especies como Calicotome villosa, Cytisus villosus, Phyllirea latifolia, Teucrium fruticans, etc. comienzan su regeneración a partir de los dos meses.

En cuanto a la respuesta adaptativa de las especies al fuego, las estrategias concretas que se observaron fueron las siguientes: a) regeneración solo por semillas $(10 \%)$; plantas que mueren tras el incendio y se regeneran de semillas preexistentes como Pinus pinaster y Cistus salvifolius. b) regeneración por yemas epicórmicas sobre el suelo (10\%); plantas cuya parte aérea resiste el calor, por adaptación de su corteza a las altas temperaturas, rebrotando mediante yemas aéreas como Quercus suber y Quercus faginea, que tambien lo hacen desde el cuello de la raíz. c) regeneración por yemas epicórmicas bajo el suelo $(46 \%)$; plantas que pierden su parte aérea pero que son capaces de rebrotar desde el cuello de la raíz o cepa (lignotuber). Es la estrategia mas frecuente en 
las especies del alcornocal y entre ellas se encuentran Viburnum tinus y Erica arborea. d) regeneración por yemas no epicórmicas bajo el suelo $(27 \%)$; plantas que poseen capacidad de regeneración vegetativa mediante yemas no epicórmicas existentes bajo el suelo como Rubus ulmifolius y Phlomis purpurea. e) sin regeneración (7\%); plantas que no se regeneran tras el incendio y que se pueden considerar como las más frágiles respecto al fuego, su presencia es un indicador de zonas no incendiadas recientemente. Es el caso de Abies pinsapo y Asplenium onopteris.

Hay que destacar que prácticamente la totalidad de las especies leñosas constituyentes del alcornocal (salvo Abies pinsapo) son capaces de regenerar su parte aérea a partir de la subterránea o de la propia parte aérea quemada, con todas las ventajas que esto conlleva frente a la regeneración por semilla. A los pocos meses del incendio se podían observar dos grupos de regeneración, el de las plántulas procedentes de semillas de especies que murieron en el incendio ( 3 especies) y el de las que regeneraron su parte aérea (el resto).

El quejigo (Quercus faginea) es capaz de rebrotar desde ramas quemadas, desde el cuello de la raíz e incluso por estolones superficiales, circunstancia que Souto y Monteiro (1987) no observaron en el quejigo de Portugal.Ulex baeticus tiene capacidad regenerativa desde yemas epicórmicas bajo el suelo, circunstancia que lo separa del Ulex parviflorus subsp. rivasgodayanus, que no presenta este tipo de regeneración y vuelve a aparecer exclusivamente mediante semillas (Carreira $e t$ al, 1991), y lo relaciona con el grupo $U$. europaeus, que forma parte de brezales y tojales caracterizados por su rebrote tras el incendio en el noroeste de España (Casal et al, 1984). Abies pinsapo no presenta ni regeneración vegetativa ni por semilla. Los pinsapos más altos con la copa no afectada por las llamas no murieron, lo cual puede indicar que su corteza les ofrece cierta protección. Arbutus unedo florece y fructifica al año del incendio.Cytisus grandiflorus aparece con gran profusión en la zona incendiada procedente de semilla. Daphne gnidium es la primera planta en mostrar actividad vegetativa tras el incendio, alcanza la máxima elongación y florece al año del incendio; estas cualidades, unidas a que no es apetecible al ganado, pueden explicar el amplio espectro fitosociológico que esta especie presenta. Phlomis purpurea florece al año del incendio. Pistacia therebintus y Pteridium aquilinum muestran elongación en la estación en que normalmente se encuentra en reposo vegetativo (Pérez Latorre et al, 1995). Quercus suber muestra gran cantidad de plántulas que podrían proceder de las que vegetan entre la hojarasca hasta que un incendio permite la llegada de luz y la fertilización del suelo. Tal y como Mesleard et al (1991) observaron, no se detectaron plántulas de Erica arborea ni de Arbutus unedo.

Nuestros datos sobre la estrategia de regeneración son coincidentes con los de García Fayos (1991) en alcornocales valencianos, aunque podemos destacar el mayor porcentaje de especies que se regeneran vegetativamente en los andaluces.

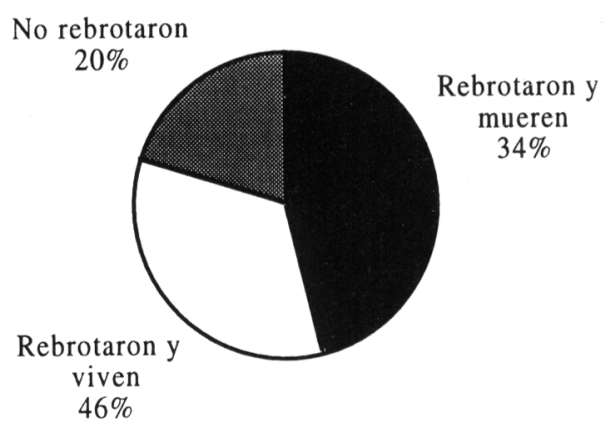

Figura 1. Porcentaje de supervivencia de Quercus suber en la zona estudiada al año del incendio. Percentage of survival in Quercus suber after one year from the fire in the study area. 


\begin{tabular}{|c|c|c|c|c|c|}
\hline \multirow[t]{2}{*}{ Especie } & \multicolumn{3}{|c|}{ Elongación en centímetros } & \multicolumn{2}{|c|}{ Tipo de regeneración } \\
\hline & Sep. & Oct. & Nov. & Dic. & \\
\hline Abies pinsapo & - & - & - & - & $\mathrm{V}$ \\
\hline Arbutus unedo & + & 5 & 40 & 42 & III(II) \\
\hline Asplenium onopters & - & - & - & - & $\mathrm{V}$ \\
\hline Calicotome villosa & - & - & - & 10 & IV \\
\hline Chamaerops humilis & - & 30 & 60 & 60 & II(IV) \\
\hline Cistus salvifolius & - & - & $\mathrm{p}$ & $\mathrm{p}$ & I(II) \\
\hline Cytisus grandiflorus & + & + & + & 15 & I(III) \\
\hline Cytisus villosus & - & - & 7 & 7 & III \\
\hline Daphne gnidium & + & 30 & 80 & 80 & III \\
\hline Erica arborea & + & + & 15 & 25 & III \\
\hline Juniperus oxycedrus & - & 2 & 10 & 20 & III(II) \\
\hline Myrtus communis & - & - & 60 & 60 & III \\
\hline Phlomis purpurea & + & + & 12 & 30 & III \\
\hline Phyllirea angustifolia & - & 5 & 25 & 40 & III \\
\hline Phyllirea latifolia & - & - & 15 & 60 & III \\
\hline Pinus pinaster & - & - & $\mathrm{p}$ & $\mathrm{p}$ & I \\
\hline Pistacia lentiscus & - & 20 & 53 & 55 & III \\
\hline Pistacia therebintus & - & 5 & 60 & 60 & III \\
\hline Pteridium aquilinum & + & + & 50 & 55 & IV \\
\hline Quercus coccifera & - & 5 & 40 & 40 & IV(III) \\
\hline Quercus faginea & $-1+$ & $10 / 30$ & $10 / 50$ & $25 / 60$ & $\mathrm{II}(\mathrm{I} / \mathrm{III})$ \\
\hline Quercus suber & $-1+$ & $10 / 30$ & $10 / 60$ & $35 / 80$ & II(I/III) \\
\hline Rosa canina & - & - & 30 & 30 & IV \\
\hline Rubia peregrina & + & + & 5 & 15 & IV \\
\hline Rubus ulmifolius & - & - & 70 & 70 & IV \\
\hline Smilax aspera & - & - & - & 80 & IV \\
\hline Teucrium fruticans & - & - & 10 & 10 & III \\
\hline Ulex baeticus & + & 5 & 15 & 15 & III \\
\hline Urginea maritima & - & 2 & 30 & 30 & IV \\
\hline Viburnum tinus & - & 15 & 15 & 35 & III \\
\hline
\end{tabular}

Tabla 2. Dinámica de la regeneración en el área de estudio (fecha del incendio: agosto de 1991). Se indica la fecha de aparición de la regeneración (mensual) y altura máxima alcanzada (en centimetros) así como el tipo de regeneración principal y secundaria (entre paréntesis): I=semilla, II=yemas epicórmicas sobre el suelo, III=yemas epicórmicas bajo el suelo, IV=yemas no epicórmicas bajo el suelo, V=sin regeneración. Para las especies de Quercus se indica la elongación de brote de cepa/brote aéreo. (-)=ausencia de regeneración, $(+)=$ inicio de regeneración, $(\mathrm{p})=$ aparición de plántula. Regeneration sequence in the study area (fire date: August 1991), indicating regeneration appearance date (monthly) and maximum reached height (in $\mathrm{cm}$.) as well as principal and secondary (in brackets) regeneration kind: $I=$ seed, $I I=$ above ground epicormic buds, III=below ground epicormic buds, IV= below ground non epicormic buds, $V=$ no regeneration. For Quercus species is indicated the growth from base shoot/branch shoot. $(-)=$ no regeneration, $(+)=$ initial regeneration, $(p)=$ seed germination appearance.

El porcentaje de regeneración de Quercus suber tras el incendio es muy alto $(80 \%)$. Sin embargo, al año del incendio, un $43 \%$ de ellos no llegan a sobrevivir, quedando al final en estado de regeneración un $46 \%$ del total. La existencia de corcho de unos 6 años ha debido influir positivamente en la regeneración del alcornoque.
En la tabla 3 se presentan los datos finales (Agosto, 1993) de las tres parcelas elegidas en el área incendiada, en cuyos datos podemos observar la posible pérdida de diversidad específica, del número de individuos y los efectos del pastoreo sobre la recuperación de la vegetación. Se detecta en las parcelas estudiadas (libre y vallada) la desaparición de 
algunas especies presentes en la parcela intacta. Destacan las pérdidas de Abies pinsapo y Asplenium onopteris que tampoco aparecen en las observaciones más amplias de la tabla 2, por lo que consideramos a éstas especies como las más frágiles frente al fuego en el alcornocal estudiado. El resto de especies desaparecidas, como Quercus faginea, Rubus ulmifolius y Smilax mauritanica, lo son sólo en las parcelas estudiadas, ya que sí hemos detectado su regeneración en el resto del areal incendiado (tab. 2).

Se detecta una pérdida importante de individuos totales, muy semejante entre las dos parcelas estudiadas (cercana al 50\%), lo que nos permite suponer que es debida al fuego y no a la acción del ganado. Entre las especies mas afectadas destacamos a Phyllirea latifolia, que casi desaparece de las dos parcelas y Viburnum tinus que disminuye su presencia de modo importante en la parcela no vallada. El aumento de individuos en algunas especies puede ser debido a un número inicial mayor a lo previsto en las parcelas quemadas en función de particularidades microtopográficas en la zona afectada por el fuego. El resto de las especies mantienen un número de individuos regenerados similar al número de individuos iniciales esperado.

En la tabla 4 se presentan los resultados de altura máxima media de los brotes mas desarrollados de todos los individuos de cada especie en las dos parcelas quemadas. El estudio muestra que la acción del ganado retarda la regeneración de la mayor parte de las especies en la parcela no vallada. La elongación registrada en Invierno es extra-estacional y por tanto mucho menor que la de PrimaveraVerano. En la parcela vallada, la elongación es constante y llega a crecimientos finales

\begin{tabular}{|c|c|c|c|c|c|c|}
\hline \multirow[b]{3}{*}{ Especies } & \multirow{2}{*}{\multicolumn{2}{|c|}{ Parcela no quemada }} & \multicolumn{4}{|c|}{ Parcelas quemadas } \\
\hline & & & \multicolumn{2}{|c|}{ No vallada } & \multicolumn{2}{|c|}{ Vallada } \\
\hline & $\%$ & $n^{\circ} \mathrm{i}$ & Ei & $\mathrm{Ri}$ & $\mathrm{Ei}$ & $\mathrm{Ri}$ \\
\hline Arbutus unedo & 9,7 & 29 & 43,5 & 32 & 11,6 & 7 \\
\hline Daphne gnidium & 0,3 & 1 & 1,5 & 2 & 0,4 & 1 \\
\hline Erica arborea & 12,1 & 36 & 54 & 87 & 14,4 & 23 \\
\hline Juniperus oxycedrus & 0,3 & 1 & 1,5 & 9 & 0,4 & 4 \\
\hline Phyllirea angustifolia & 2,3 & 7 & 10,5 & 8 & 2,8 & 4 \\
\hline Phyllirea latifolia & 18,5 & 55 & 82,5 & 7 & 22 & 1 \\
\hline Quercus faginea (a) & 2,3 & 7 & 10,5 & 0 & 2,8 & 0 \\
\hline Quercus suber (a) & 1 & 3 & 4,5 & 4 & 1,2 & 3 \\
\hline Rubia peregrina & 11,7 & 35 & 52,5 & 69 & 14 & 7 \\
\hline Viburnum tinus & 17,4 & 52 & 78 & 29 & 20,8 & 13 \\
\hline Asplenium onopteris & 20,5 & 61 & 91,5 & 0 & 24,4 & 0 \\
\hline Rubus ulmifolius & 1,7 & 5 & 7,5 & 0 & 2 & 0 \\
\hline Smilax mauritanica & 0,7 & 2 & 3 & 0 & 0,8 & 0 \\
\hline Abies pinsapo & 0,3 & 1 & 1,5 & 0 & 0,4 & 0 \\
\hline Pistacia terebinthus & 1,0 & 3 & 4,5 & 2 & 1,2 & 0 \\
\hline Total de individuos & 100 & 298 & 447 & 249 & 119,2 & 63 \\
\hline Pérdida de individuos & Ei-Ri & & 198 & 14\%) & $56,2(4$ & $7 \%)$ \\
\hline
\end{tabular}

Tabla 3. Situación de las tres parcelas en Agosto de 1993. $\mathrm{n}^{\circ} \mathrm{i}=$ número de individuos por especie en la parcela intacta. Ei=individuos estimados en las parcelas quemadas. $\mathrm{Ri}=$ individuos regenerados en las parcelas quemadas. State of the three studied plots in August 1993. $n^{\circ} i=$ number of individuals by species in the non burned plot. Ei=rated individuals in the burned plots. Ri=regenerated individuals in the burned plots. 
superiores que los de la no vallada. Se ha detectado, en la parcela no vallada, una menor presión del ganado sobre los arbustos rebrotadores con el desarrollo primaveral del pastizal. Inicialmente, en la parcela no vallada, hay especies que responden al ramoneo aumentando el número de brotes en perjuicio del crecimiento de los mismos, por lo que descendiende la altura media en especies como Arbutus unedo, Phyllirea latifolia y Viburnum tinus; otras, sin ser ramoneadas, pueden mantener estable su altura (Erica arborea, Juniperus oxycedrus, Phyllirea angustifolia y Quercus sp.) o presentar un crecimiento significativo (Daphne gnidium y Rubia peregrina). En Abril-Agosto existe, en ambas parcelas, un gran incremento de la altura máxima media en todas las especies coincidiendo con la estación de máximo desarrollo vegetativo (Pérez Latorre et al, 1995). En la parcela vallada existen dos comportamientos iniciales, especies que, debido a una regeneración escalonada de sus individuos, presentan un descenso inicial de la altura media (Phyllirea angustifolia y Quercus faginea) y especies que, debido a una regeneración sincronizada, aumentan su altura media constantemente. A partir de Abril se registra una gran elongación en ambas parcelas debida al crecimiento vegetativo estacional normal de la comunidad. Las especies mas apetecidas por el ganado (todos los brotes comidos) son Phyllirea angustifolia y Phyllirea latifolia; Arbutus unedo, Erica arborea y Viburnum tinus presentan un indice menor de ramoneo; Juniperus oxycedrus, Rubia peregrina y los Quercus en fase juvenil son poco ramoneados y Daphne gnidium no es comido en ninguna ocasión.

En la figura 1 se puede observar la incidencia que el pastoreo tiene en la parcela no vallada, esta incidencia se manifiesta en la diferencia mensual entre la regeneración global de la parcela vallada y la no vallada, referida a la suma de las alturas máximas medias del total de los individuos de todas las especies. El alcornocal alcanza en la parcela vallada elongaciones mayores que en la parcela no vallada, esta diferencia en la regeneración se produce fundamentalmente a partir de Marzo.

\begin{tabular}{lcccccccccc}
\hline & \multicolumn{4}{c}{ Parcela no vallada } & \multicolumn{5}{c}{ Parcela vallada } \\
\hline Especies & Ene & Feb & Mar & May & Ago & Ene & Feb & Mar & May & Ago \\
\hline Arbutus unedo & 10,9 & 8,5 & 7,1 & 26,4 & 49,6 & 17,6 & 17,3 & 22,3 & 69 & 139,1 \\
Daphne gnidium & 20 & 21 & 23 & 22 & 22 & 39 & 40 & 43 & 53 & 59 \\
Erica arborea & 4 & 3,8 & 5,3 & 24 & 33 & 4,9 & 6,7 & 9,1 & 30,7 & 54,3 \\
Juniperus oxycedrus & 2,8 & 3,1 & 3,2 & 15,8 & 22,1 & 3,5 & 4,3 & 6,7 & 20 & 29 \\
Phyllirea angustifolia & 13,1 & 10,2 & 10,3 & 11,3 & 57,8 & 10,8 & 8,3 & 8,0 & 37,8 & 68,3 \\
Phyllirea latifolia & 8,7 & 9,2 & 4,6 & 34,2 & 36,0 & 3 & 4 & 7 & 34,5 & 50 \\
Quercus faginea (p) & 6,1 & 5,4 & 6,1 & 22,5 & 26,3 & 7,7 & 10,5 & 7,0 & 51,7 & 58,0 \\
Quercus suber (p) & 5,4 & 5,4 & 4,8 & 14,8 & 15,9 & 3,5 & 4,5 & 5,0 & 16,0 & 14,3 \\
Rubia peregrina & 5,6 & 5,4 & 6,8 & 12,4 & 12,0 & 4 & 4,8 & 7,7 & 17,8 & 18,3 \\
Viburnum tinus & 13,8 & 8,7 & 7,5 & 20,9 & 24,5 & 12,7 & 12,1 & 15,8 & 35,0 & 40,4 \\
\hline Totales & 90,3 & 80,8 & 112,5 & 240,1 & 313,2 & 106,7 & 112,4 & 131,5 & 365,5 & 516,3 \\
\hline
\end{tabular}

Tabla 4. Altura máxima media (en cm.) comparada entre la parcela vallada y la no vallada de Enero a Agosto de 1993. En los Quercus (p)=plántula. Maximum height average (in $\mathrm{cm}$.) between the fenced and the non fenced plots from January to August 1993. For Quercus species $(p)=y o u n g$ plant. 


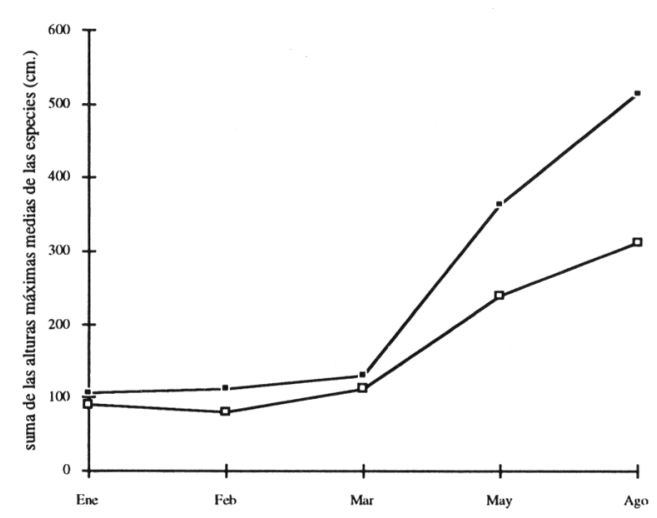

Figura1. Comparación de la suma de las alturas máximas medias del total de los individuos de todas las especies estudiadas entre la parcela vallada (línea superior) y no vallada (línea inferior). Comparison between the fenced (upper line) and non fenced (lower line) plots refered to the sum of the maximum height average of all individuals of all studied species.

Los estadíos fisiognómicos por los que pasa el área quemada, tras un año de observación, comienzan con un rápido desarrollo de los brotes aéreos y basales de Quercus suber, Quercus faginea, Daphne gnidium y Chamaerops humilis. Tras las primeras lluvias aparecen plántulas con escasa cobertura total y rebrotan gran cantidad de arbustos como Erica, Arbutus, Viburnum, Quercus coccifera y Phyllirea spp., y comienzan a secarse algunos brotes aéreos de alcornoque. Después de una parada invernal de crecimiento, comienza a desarrollarse el pastizal secándose mas brotes aéreos de los alcornoques, incluso mueren árboles enteros; aparecen nuevos brotes de Erica, Viburnum y Arbutus, en cepas que no habían mostrado actividad anteriormente, así como especies rizomatosas como Smilax mauritanica. La comunidad incendiada muestra su máximo desarrollo vegetativo y de floración (sólo el pastizal) en Mayo. Tras el agostamiento del pastizal anual, cubren el suelo casi en su totalidad plántulas de Cistus sp. y Halimium sp., dominando fisiognómicamente Cistus salvifolius. Los alcornoques que han sobrevivido, muestran una intensa regeneración aérea, estructurando de nuevo sus copas dando sombra y cobertura al suelo. Pasado un año del incendio, el aspecto fisiognómico del alcornocal incendiado está caracterizado por un estrato arbóreo de alcornoques, regenerados aproximadamente en un $50 \%$, otro arbustivo caracterizado por una regeneración fundamentalmente desde la base del tronco y por último un estrato herbáceo dominado por plántulas de Cistus salvifolius, Cistus ladanifer, Adenocarpus telonensis y Cytisus grandiflorus.

\section{CONCLUSIONES}

El alcornocal estudiado es una formación pirófita capaz de autoregenerarse sin necesidad de actuaciones de tipo forestal. El efecto del pastoreo sobre el alcornocal se traduce en una pérdida de biomasa reflejada en la disminución de la altura máxima media de los brotes, ralentizándose la regeneración y reestructuración de la masa boscosa. El fuego afecta negativamente al alcornocal en cuanto a la pérdida de individuos, en un grado próximo al $50 \%$. En cuanto a la pérdida de biodiversidad específica, el fuego afecta sensiblemente al alcornocal puesto que desaparecen especies como Asplenium onopteris y Abies pinsapo, siendo gravemente afectada la supervivencia de Phyllirea latifolia; la presencia de dichas especies podría considerarse como indicadora de zonas con escasa recurrencia de incendios. En las condiciones del estudio, la supervivencia de Quercus suber se aproxima al 50\%, siendo muy importante el porcentaje de alcornoques que rebrotan inicialmente pero se secan a los pocos meses.

\section{BIBLIOGRAFÍA}

ARIANOUTSON, M. \& N. S. MARGARIS -1981Early stages of regeneration after fire in a phryganic ecosystem (East Mediterranean) I. 
Regeneration by seed germination. BiologieEcologie Méditerranéenne, 8:119-128.

CARREIRA, J., NIELL, F. J. y ASENSI, A. -1991Sustitución de comunidades y concentración de nutrientes en plantas tras incendios forestales en una cuenca subárida del S.E. de España. Orsis, 6:199-214.

CASAL, U., BASANTA, M. y GARCÍA NOVO, F. -1984- La regeneración de los montes incendiados en Galicia. Monografía 99. Universidad de Santiago de Compostela.

DELARZE, R., CALDELARI, D. \& HAINARD, P. -1992- Effects of fire on forest dynamics in southern Switzerland. Journal of Vegetation Science, 3:55-60.

GARCÍA FAYOS, P. - 1991- La vegetación silicícola de la Sierra Calderona (Comunidad Valenciana). Lazaroa, 12:317-322.

LE HOUEROU, H. N. -1981-Impact of man and his animals on mediterranean vegetation. In: $\mathrm{Di}$ Castri, F., Goodall, D. W. \& Specht, R. L. (eds.) Ecosystems of the world 11: Mediterranean type shrublands. Amsterdam.

MESLEARD, F. \& J. LEPART -1991-Germination and seedling dynamics of Arbutus unedo and Erica arborea on Corsica. Journal of Veg. Science, 2:155-164.

NAVEH, Z. -1974- Effects of fire in the Mediterranean region. In: Kozlowsky \& Algren (eds.) Fire and Ecosystems. Academic Pres. New York. pp. 401-434.

NAVEH, Z. -1991- The role of fire in mediterranean vegetation. Botanika Chronika, 10:385-405.

NIETO CALDERA, J. M., A. V. PÉREZ LATORRE y B. CABEZUDO -1991- Biogeografía y series de vegetación de la provincia de Málaga (España). Act. Bot. Malacitana, 16(2):417-436.

ORSHAN, G. -1986- Plant form as describing vegetation and expressing adaptation to environment. Anali di Botanica, 54: 7-37.

PÉREZ LATORRE, A. V., J. M. NIETO CALDERA y B.CABEZUDO -1993- Contribución al conocimiento de la vegetación de Andalucía. II. Los alcornocales. Acta Bot. Malacitana, 18: 223-258.

PÉREZ LATORRE, A. V., J. M. NIETO CALDERA y B.CABEZUDO -1994- Contribución al conocimiento de la vegetación de Andalucía. III. Series de vegetación caracterizadas por Quercus suber L. Acta Bot. Malacitana, 19: 169-183.
PÉREZ LATORRE, A. V., B. CABEZUDO, J. M. NIETO CALDERA y T. NAVARRO -1995Caracterización ecomorfológica y fenomorfológica de alcornocales andaluces (Málaga, España). Anales. Jard. Bot. Madrid (en prensa).

PERY, M. -1989- Observacions sobre la regeneració de les suredes després del foc. Scientia Gerundensis, 15:181-184.

PONS, A. \& M. THINON -1987- The role of fire from palaeoecological data. Ecol. Med., 13(4):311.

SOUTO, C. \& A. MONTEIRO -1987- Ecological fire influences on Q. suber forest ecosystems. Ecologia Mediterranea, 13(4):69-77.

TRABAUD, L. -1987- Dynamics after fire of sclerophyllous plant communities in the Mediterranean basin. Ecol. Med., 13(4): 25-37.

Aceptado para su publicación en Enero de 1995

Dirección de los autores. Departamento de Biología Vegetal. Facultad de Ciencias. Universidad de Málaga. Campus de Teatinos. Apdo. 59. 29080, Málaga. Fax (95) 2131944. 